\title{
RIPK1 prevents TRADD-driven, but TNFR1 independent, apoptosis during development
}

\author{
Holly Anderton ${ }^{1,2}$. Esther Bandala-Sanchez ${ }^{1,2}$ - Daniel S Simpson ${ }^{1,2} \cdot$ James A Rickard ${ }^{1,2} \cdot$ Ashley P Ng (1),2 \\ Ladina Di Rago ${ }^{1,2} \cdot$ Cathrine Hall ${ }^{1,2} \cdot$ James E Vince ${ }^{1,2} \cdot$ John Silke $\mathbb{D}^{1,2} \cdot$ Gianmaria Liccardi $^{3} \cdot$ Rebecca Feltham ${ }^{1,2}$
}

Received: 21 December 2017 / Revised: 5 June 2018 / Accepted: 5 July 2018 / Published online: 5 September 2018

(c) ADMC Associazione Differenziamento e Morte Cellulare 2018

\begin{abstract}
RIPK1 is an essential downstream component of many pattern recognition and death receptors. RIPK1 can promote the activation of caspase-8 induced apoptosis and RIPK3-MLKL-mediated necroptosis, however, during development RIPK1 limits both forms of cell death. Accordingly, Ripk $^{-{ }^{--}}$mice present with systemic cell death and consequent multi-organ inflammation, which is driven through the activation of both FADD-caspase-8 and RIPK3-MLKL signaling pathways causing perinatal lethality. TRADD is a death domain (DD) containing molecule that mediates signaling downstream of TNFR 1 and the TLRs. Following the disassembly of the upstream receptor complexes either RIPK1 or TRADD can form a complex with FADD-caspase-8-cFLIP, via DD-DD interactions with FADD, facilitating the activation of caspase-8. We show that genetic deletion of Ripk1 licenses TRADD to complex with FADD-caspase- 8 and activates caspase-8 during development. Deletion of Tradd provided no survival advantage to Ripk ${ }^{-1-}$ animals and yet was sufficient to reduce the systemic cell death and inflammation, rescue the intestinal and thymic histopathologies, reduce cleaved caspases in most tissues and rescue the anemia observed in $R i p k 1^{-/-}$neonates. Furthermore, deletion of Ripk 3 is sufficient to rescue the neonatal lethality of Ripk $^{-/-} \mathrm{Tradd}^{-/-}$animals and delays but does not completely prevent early mortality. Although Ripk3 deletion provides a significant survival advantage, $\operatorname{Ripk1}^{-/-} \mathrm{Tradd}^{-{ }_{-}}$Ripk $^{-/-}$animals die between 22 and 49 days, are runty compared to littermate controls and present with splenomegaly. These findings reveal a new mechanism by which RIPK1 limits apoptosis through blocking TRADD recruitment to FADD and preventing aberrant activation of caspase-8.
\end{abstract}

\section{Introduction}

These authors contributed equally: Gianmaria Liccardi, Rebecca Feltham.

Electronic supplementary material The online version of this article (https://doi.org/10.1038/s41418-018-0166-8) contains supplementary material, which is available to authorized users.

Gianmaria Liccardi

gianmaria.liccardi@icr.ac.uk

$\triangle$ Rebecca Feltham

feltham.r@wehi.edu.au

1 The Walter and Eliza Hall Institute for Medical Research, 1G Royal Parade, Parkville, Melbourne, VIC 3050, Australia

2 Department of Medical Biology, University of Melbourne, Parkville, VIC 3050, Australia

3 Breast Cancer Now Toby Robins Research Centre, Institute of Cancer Research, Mary-Jean Mitchell Green Building, Chester Beatty Laboratories, Fulham Road, London SW3 6JB, UK
Receptor interacting serine protein kinase 1 (RIPK1) is a protein that harbors both kinase activities and scaffolding roles, and is involved in a number of signaling pathways that regulate the events downstream of Tumor necrosis factor receptor 1 (TNFR1), Toll-like receptors (TLRs), Retinoic acid-inducible gene I (RIG-I) and Interferons [1-5]. Genetic studies in mice have demonstrated the importance of RIPK1 in the maintenance of tissue homeostasis, revealing that RIPK1 is an essential inhibitor of both apoptotic and necroptotic cell death during development [6-10]. Ripkl knockout mice present with severe multi-organ inflammation and systemic cell death, which causes lethality of RipkI $^{-1-}$ animals late in gestation or shortly after birth $[6-8,11]$. This lethality is rescued by codeletion of caspase- 8 and Ripk 3 leading to the survival of these animals until adulthood [6-8]. The numerous Ripk1 ${ }^{-/}$induced pathologies observed in these animals are in 
part due to the activation of both TNFR1 and Myeloid differentiation primary response 88 (Myd88) driven pathways [6, 8]. Deletion of Tnfrl is sufficient to ameliorate large intestinal damage in Ripk $1^{-/}$animals and, depending on the background, Ripk $1^{-/-}$Tnfr $1^{-/-}$mice have delayed mortality $[6,12]$ or are provided no protection against the Ripkl knockout induced perinatal lethality or the systemic inflammation [8]. On the other hand, co-deletion of $M y d 88$ reduces the systematic inflammation allowing mice to survive to P4 [8]. Deletion of the necroptotic effectors Receptor interacting protein kinase 3 (RIPK3) or Mixed Lineage Kinase Domain Like Pseudokinase (MLKL) affords protection against Ripk $^{-/-}$induced systemic inflammation, skin hyperplasia and anemia, while providing no protection against the caspase- 8 driven intestinal damage [8]. Collectively these genetic studies demonstrate that RIPK1 inhibits both Fas-associated protein with death domain (FADD)-caspase-8 driven apoptosis and RIPK3mediated necroptosis during development. Consistently, in various cellular settings, the activation of both apoptotic and necroptotic cell death pathways can be RIPK1 independent [13-17]. Activation of RIPK3 in the absence of RIPK1 during development has recently been shown to be dependent on the protein DNA dependent activator of Interferon regulatory factors (DAI), whereby the RIP homotypic interaction motif (RHIM) domain of RIPK1 prevents the association of DAI with RIPK3, limiting RIPK3 activation $[18,19]$. On the other hand, the mechanism leading to the activation of the FADD-caspase- 8 axis in the absence of RIPK1 during development has not been described.

Downstream of TNFR1, survival-signaling pathways such as $\mathrm{NF}-\kappa \mathrm{B}$ are initially activated from a primary signaling complex (complex-I) to prevent the activation of caspase- 8 through the up-regulation of inhibitory proteins such as cFLIP. Removal of the cellular inhibitor of apoptosis proteins (cIAPs) [20-22], inhibition of MAP kinaseactivated protein kinase 2 (MK2) [23-27], inhibition of Transforming growth factor $\beta$-activated kinase 1 (TAK1) [28] or inhibition of protein synthesis using cycloheximide [29, 30] leads to cell death which is driven through the formation of secondary complexes containing either Tumor necrosis factor receptor type 1-associated death domain protein (TRADD)-FADD-cFLIP-caspase-8 (complex-IIa), RIPK1-FADD-cFLIP-caspase-8 (complex-IIb) or RIPK3MLKL (complex-IIc/necrosome) [31, 32] depending on the insult.

Given that both TRADD and RIPK1 contain a death domain and both can interact directly with FADD to drive caspase- 8 activation in cells, we hypothesized that the activation of caspase- 8 in the absence of RIPK 1 in vivo was TRADD-mediated. We now demonstrate that although $\operatorname{Ripk}^{-/-}$Tradd $^{-/-}$animals still die perinatally, they show a full rescue of the caspase-8-driven intestinal and thymic phenotypes and an overall reduction in the cleavage of caspases within multiple tissues. Ripk ${ }^{-/-}$Tradd $^{-/-}$mice still exhibit systemic inflammation and skin hyperplasia, presumably due to RIPK3/MLKL activation, but show significantly reduced amounts of systemic apoptosis and are no longer anemic. Deletion of Ripk3 provided significant, albeit incomplete, survival advantage to Ripk $^{-/-}$Tradd $^{-/-}$mice, which interestingly highlights clear differences between $\operatorname{Ripk}^{-/-}$Tradd $^{-1-}$ Ripk3 $^{-/-}$animals and Ripk1 ${ }^{-/}$Casp- $8^{-/-}$Ripk3 ${ }^{-/-}$animals.

\section{Results}

\section{Tradd deficiency prevents Ripk ${ }^{-/-}$induced pathology in multiple organs but provides no survival advantage to Ripk $^{-/-}$neonates}

To assess the role of TRADD in the cell death and inflammation driven pathologies induced by Ripkl deletion we generated RipkI ${ }^{-/}$Tradd $^{-1-}$ mice and found that co-deletion of Tradd provides no survival advantage to Ripk $1^{-/-}$neonates (Fig. 1a, b) with Ripk1 $1^{-/-}$Tradd $^{-/-}$pups typically being found dead at, or shortly after, birth. We did, however, observe that loss of Tradd completely rescued the large intestinal phenotype of Ripk1 $1^{-/}$mice; characterized by a lack of goblet cells, a shortening of crypt lengths and a sloughing of intestinal cells into the lumen (Fig. 1c-e and S1). In addition, these animals show a clear rescue of the thymic histopathology characteristic of $\mathrm{Ripk1}^{-/-}$animals, as previously described [8, 11] (Fig. 1c and S1). Tradd deficiency did not ameliorate the Ripk1 ${ }^{-/-}$induced epidermal hyperplasia and resulted in only a modest decrease in Keratin-6 staining, a wound-induced gene which is usually confined to hair follicles in healthy skin but is highly expressed throughout the epidermis of Ripkl ${ }^{-/-}$mice. The significant amelioration of the Ripk1 $1^{-1-}$-induced histopathologies seen upon co-deletion of Tradd closely mimicked the rescue seen in Ripk1 $^{-/-}$Tnfrl $1^{-/-}$neonates (Fig. 1c), but like the Tnfrl deletion provided no survival advantage to Ripk $^{-/-}$neonates on a C57BL/6 background [8].

\section{Ripk1 ${ }^{-/-}$anemia, but not the increase in white blood cell production, is rescued by co-deletion of tradd}

Ripk $1^{-/-}$mice have severe anemia and neutrophilia, with a massive increase in circulating inflammatory cytokines and chemokines detectable within the plasma of these animals [8, 33]. Interestingly, co-deletion of Tradd is sufficient to prevent the anemia but not the increase in white blood cell production (Fig. 2a and S2A). Normal numbers of red blood cells in Ripk $^{-/-}$Tradd $^{-/-}$animals correlates with a 
A

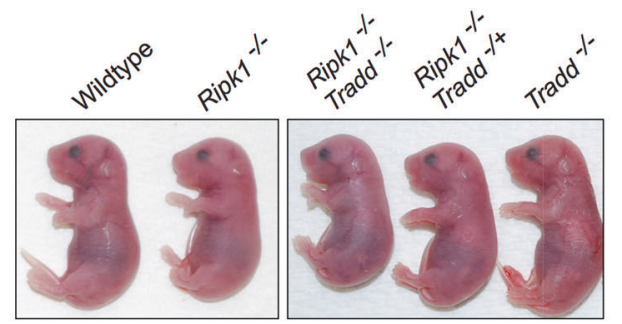

\begin{tabular}{|c|c|c|c|}
\hline B & Tradd $^{/-}$Ripk1 $^{+/+}$ & $\operatorname{Tradd}^{-/-}$Ripk1 $^{+/-}$ & Tradd ${ }^{/-}$Ripk $^{-1 /}$ \\
\hline \multirow{2}{*}{18.5} & $13(13)$ & $29(25)$ & $8(13)$ \\
\hline & $\% \quad 26(25)$ & $58(50)$ & $16(25)$ \\
\hline \multirow{2}{*}{ Po } & $\# \quad 60(52)$ & \# $\quad 119(104)$ & $29(52)$ \\
\hline & $\% \quad 29(25)$ & $57(50)$ & $14(25)$ \\
\hline \multirow{2}{*}{ P1 } & \# $\quad 49$ (42) & \# 104 (84) & $14(42)$ \\
\hline & $\% \quad 29(25)$ & $\% \quad 62(50)$ & $8(25)$ \\
\hline \multirow{2}{*}{ P21 } & \# 31 (26) & $\# \quad 74(53)$ & $0(26)$ \\
\hline & $\% 30(25)$ & $\% 70(50)$ & $0(25)$ \\
\hline Total & $\# 153$ & $\# 326$ & $\# 51$ \\
\hline
\end{tabular}

C

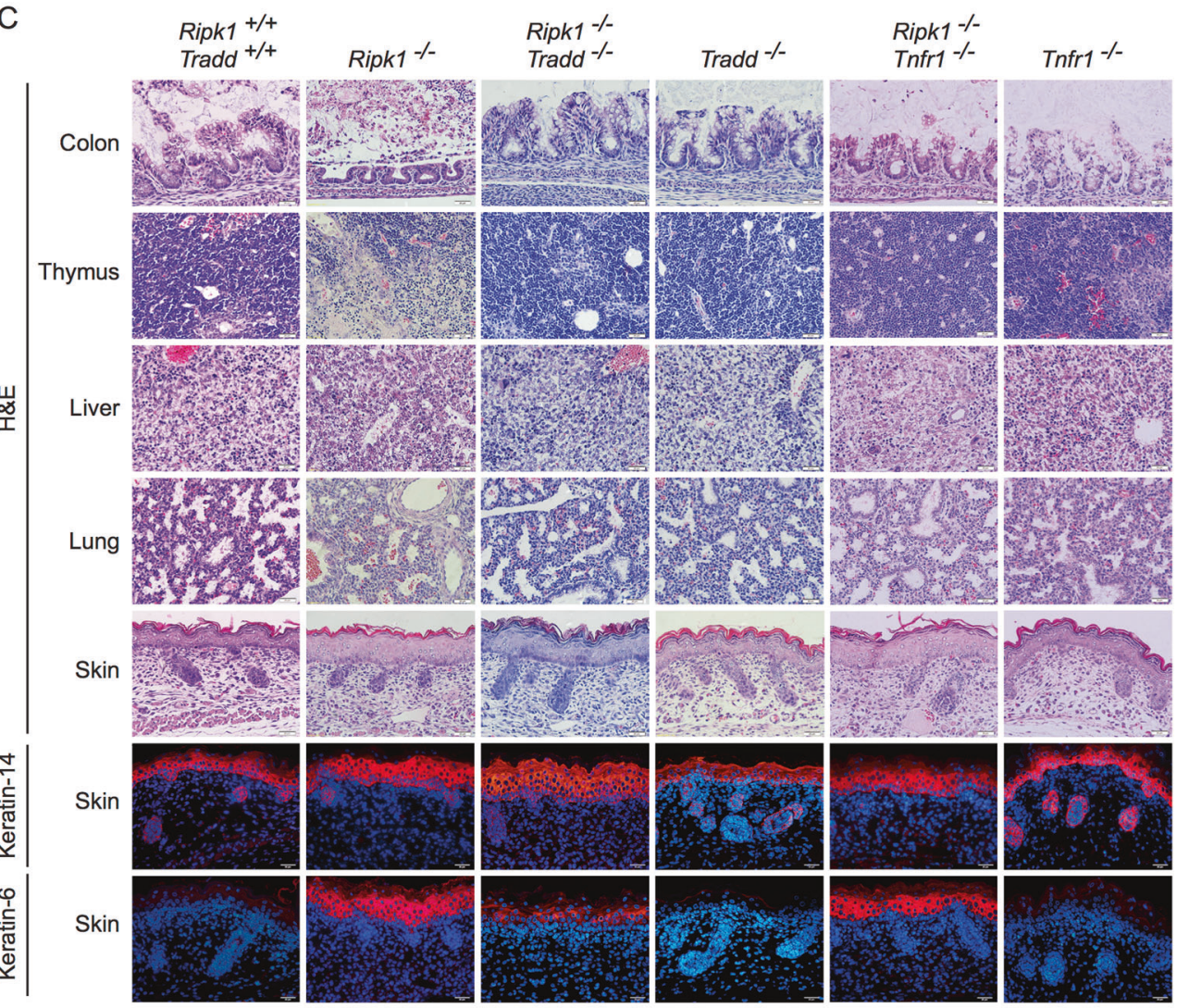

D Goblet Cells in Large Intestine

E
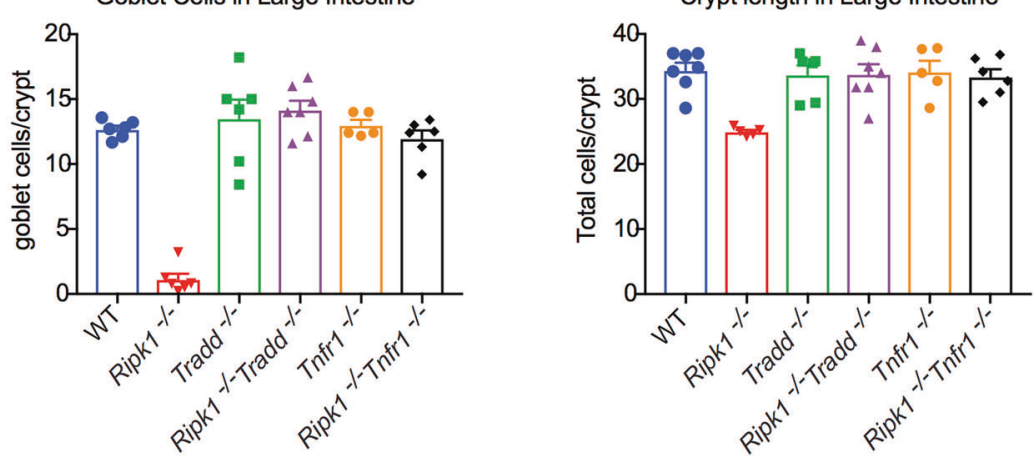

Fig. 1 Tradd Deficiency Provides No Survival Advantage to Ripk1 ${ }^{-1-}$ Neonates but Prevents Multiple Organ Histopathologies. a E18.5 neonates of indicated genotypes. b Survival numbers (\#) and percentages (\%) from E18.5 until P21 for the indicated genotypes. The table illustrates the actual numbers obtained and the expected numbers (in brackets). c Tissue sections of the indicated genotypes stained with hematoxylin and eosin (H\&E), anti-keratin-6, or anti-keratin-14 (Red). Nuclei in immunofluorescence sections were counter stained with DAPI (blue). Each image is representative of results from at least three mice. d, e Quantification of goblet cells and crypt length in the large intestine. Goblet cells were counted via $\mathrm{H} \& \mathrm{E}$ as large round white/clear cells residing within the intestinal mucosa layer. Each data point represents the average of a minimum of 5 (up to 10 where possible) "areas" from one mouse. For the large intestine an area is a single crypt from center of the crypt base to center of the next crypt base 

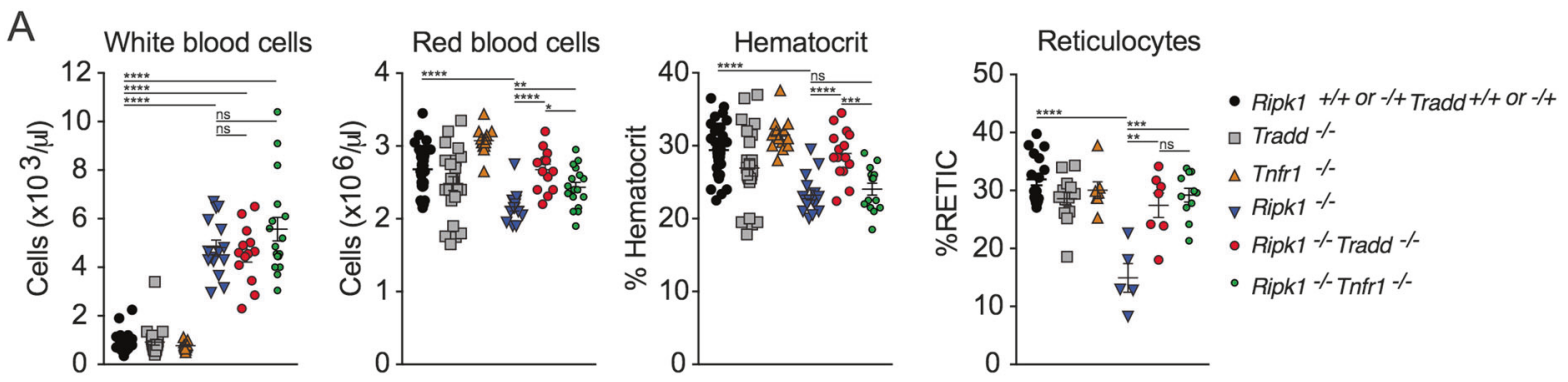

B

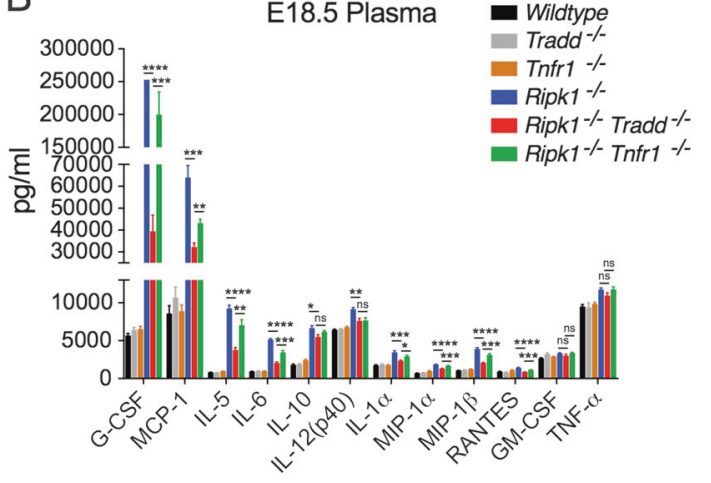

C

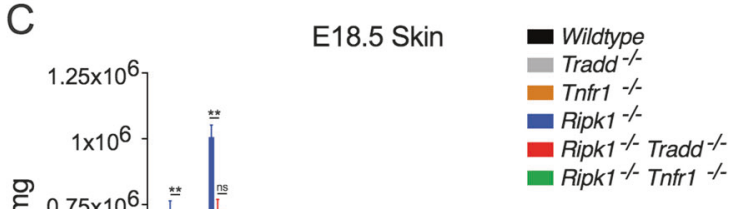

D

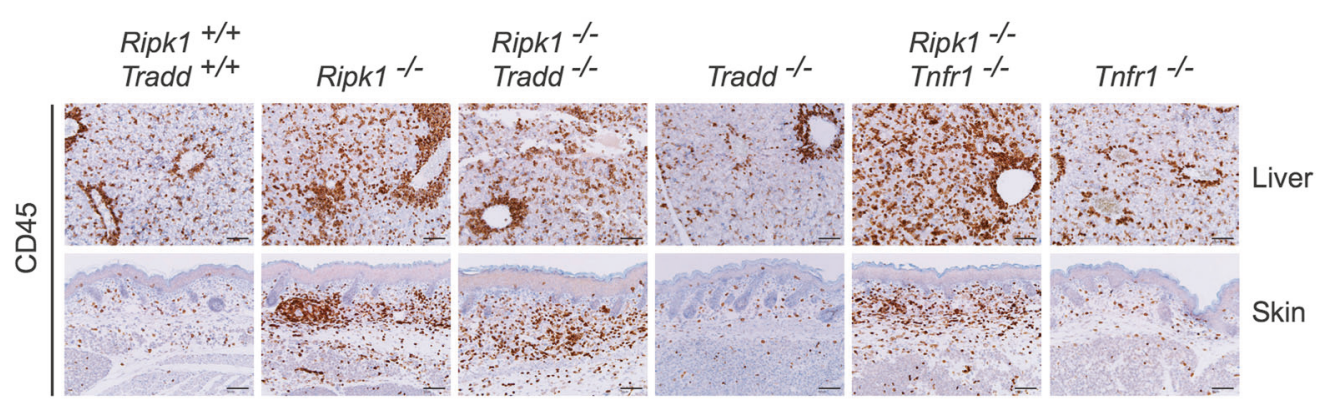

$E$

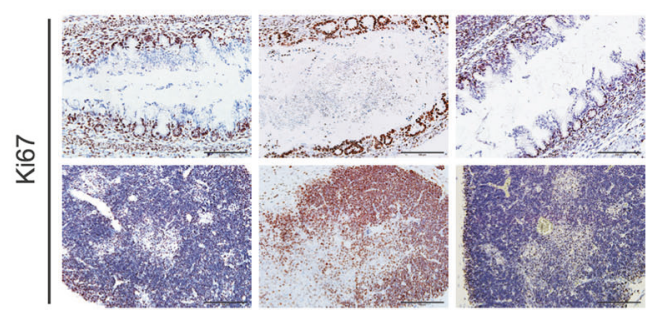

Fig. 2 Ripk $^{-1-}$ Driven Anemia and Systematic Inflammation
Requires TRADD. a Blood cells quantified with an ADVIA from E18.5 embryos $n \geq 5$. b, c Plasma, $n=7$ (b) and Skin, $n=5$ (c) cytokine levels assayed using Bioplex. All of the values for G-CSF within the plasma were above the reference range and were assigned a

restoration of reticulocytes measured via ADVIA (Fig. 2a) and re-confirmed by blood smear analysis of immature red blood cells (Figure S2A and S2D). We previously reported that Ripk $1^{-/-} \mathrm{Myd} 88^{-/-}$mice have a normal hematocrit and a marked reduction in the neutrophilia seen in Ripkl $^{-1-}$ mice [8]. On the other hand, Ripk1 ${ }^{-1-} \operatorname{Tnfrl}^{-/-}$animals have normal reticulocyte numbers yet still present with anemia; characterized by a reduction in total red blood cells and \% hematocrit (Fig. 2a). value of the highest standard. d, e Tissue sections of the indicated genotypes stained with CD45 (d) or Ki67 (e) (brown) and counterstained with hematoxylin (blue). Each image is representative of results from at least three mice. $*=0.01-0.05, * *=0.001-0.01, * * *$ $=0.0001-0.001, * * * *=<0.0001, \mathrm{~ns}=\geq 0.05$

\section{Tradd deficiency reduces the systemic inflammation} and hyperproliferation observed in Ripk $1^{-/-}$mice

Cytokines in the plasma and skin of Ripk1 ${ }^{-/-}$Tradd $^{-/-}$ animals were significantly reduced compared to Ripk1 ${ }^{-/-}$ mice, albeit still higher than their wild type counterparts (Fig. 2b, c, S2B and S2C). In the plasma, Tradd deletion resulted in a stronger reduction in a subset of cytokines than Tnfrl deletion (Fig. 2b), suggesting a role for TRADD 
independent of TNFR1 signaling. Consistent with the skin phenotypes, the level of leukocyte infiltration $(\mathrm{CD} 45+)$ within the livers and skins of $\mathrm{RipkI}^{-/} \mathrm{Tradd}^{-/}$mice (E18.5) was unchanged when compared to Ripkl ${ }^{-/}$or Ripk $1^{-1-}$ Tnfrl $^{-1-}$ mice (Fig. 2d). There were no notable differences in CD11b positive cells between any of the genotypes within the liver and skin (Figure S2E) or in a panel of other tissue sections (data not shown). Ki67 staining showed hyper proliferation through the entire crypt length and thymic lobes of $R_{i p k I^{-/}}$mice, while RipkI $^{-/-}$Tradd $^{-/-}$, and consistently the RipkI ${ }^{-/-} \mathrm{Tnfrl}^{-/-}$, present a Ki67 staining pattern concordant with normal thymic and colonic structure (Fig. 2f). No observable differences in Ki67 staining were observed in a panel of other tissues (Figure S2F).

\section{Tradd deficiency prevents caspase-3 activation in multiple Ripk ${ }^{-/-}$tissues}

Cell death driven through the activation of caspase- 8 and caspase- 3 is a key event in the disruption of multiple tissues within Ripk1 ${ }^{-1-}$ animals [6-8]. Importantly, Tradd deletion reduced the activation of caspase-3 reflected by a reduction in the number of cells with cleaved caspase- 3 in the colon, thymus and small intestine (Fig. 3a, b). The reduction in cleaved caspase- 3 positive cells across several tissues in the absence of Tradd mimicked reductions seen in Ripkl ${ }^{-/} \mathrm{Tnfr}^{-/-}$animals, suggesting that the TNFR1-TRADD axis is required for cell death during development in the absence of RIPK1 (Fig. 3a and S3A). However, when we analyzed levels of cleaved caspase- 3 within the skin and the bone marrow of RipkI $^{-1-}$ Tradd $^{-1-}$ animals we observed no reduction in cleaved caspase-3 staining via immuno-histochemistry, demonstrating that caspase- 3 can be activated via TRADD independent mechanisms in the skin of Ripkl ${ }^{-/}$animals (Fig. 3a and S3B). Notably within the plasma of Ripk $1^{-/-}$neonates we saw that RipkI ${ }^{-/-}$Tradd $^{-/-}$mice had

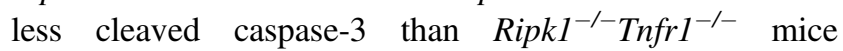
(Fig. 3c). Taken together our results suggest that the TNFR1-TRADD signaling pathway is central to the multiorgan pathologies seen in Ripkl ${ }^{-/-}$animals but that TRADD is also involved in signaling pathways outside of TNFR1.

\section{Cell death and inflammatory markers are altered in a tissue specific manner upon deletion of tradd from Ripk1 $^{-1-}$ neonates}

In order to obtain a detailed insight into the effects of Tradd deletion in both apoptotic and necroptotic tissues, we assayed known markers of these signaling pathways in whole tissues dissected from E18.5 embryos (Figure S4A and S4B) via Western blot. Consistent with our histological analysis (Figs. 1c and 3a and S3A), cleaved caspase- 8 was markedly reduced in cell extracts from the colons and thymi (Fig. 4a and S4C). In the skin, where lack of TRADD did not provide significant protection, there was a modest reduction in levels of cleaved caspase-8 when compared to Ripk1 ${ }^{-/-}$mice (Fig. 4b). Unexpectedly, there was a clear decrease in the levels of cleaved caspase-3 within the skin lysates of Ripk $^{-1-}$ Tradd $^{-1-}$ mice (Fig. 4b). The epidermal skin phenotype in Ripk1 $^{-/-}$mice is RIPK3-MLKL mediated [8], thus the heightened levels of cleaved caspases in the skin lysates of Ripkl $^{-/-}$nenonates is likely representative of cleaved caspases within the dermis. Levels of RIPK3 and MLKL were increased in the colons of Ripk1 ${ }^{-/-}$mice compared with wild type mice, and there were only slight differences in the total levels of RIPK3 and MLKL in Ripk $^{-/-}$Tradd $^{-/-}$tissues (Fig. 4a, b). These differences did not correlate with the levels of the inflammatory DAMP IL-33, which appeared unchanged in the colons and clearly reduced in the skin and thymi lysates of Ripkl ${ }^{-/}$Tradd $^{-/-}$animals. It has been previously demonstrated that in Ripk1 $^{-/-}$embryos the levels of TRAF2 and cIAP1 are decreased in response to exogenous TNF [34]. The levels of cIAP1 and TRAF2 were, however, unchanged in unstimulated tissues dissected across all genotypes. We could not reliably detect cFLIP within the colons of our E18.5 embryos; however, we did observe what appeared to be a small fragment of cFLIP, in the skin that occurred exclusively in genotypes where Ripkl was deleted, which correlated with a reduction in $\operatorname{cFLIP}_{\mathrm{L}}$ but which appeared unchanged in Ripk1 ${ }^{-/}$Tradd $^{-/-}$animals (Fig. 4b).

\section{Caspase-8 activation in Ripk ${ }^{-/-}$neonates is driven via a TRADD-FADD complex}

The reduction in cleaved caspase-8 in RipkI $^{-/-}$Tradd $^{-/-}$ animals suggests that TRADD contributes to the activation of caspase-8 in Ripkl deficient mice. Despite similarities with Ripk1 ${ }^{-/}$Tnfrl ${ }^{-/-}$animals, RipkI ${ }^{-/-}$Tradd $^{-/}$mice also display distinct phenotypic differences. To determine whether TRADD can indeed directly interact with a FADDcaspase- 8 containing complex in the absence of RIPK1, we employed the proximity ligation assay (PLA). This is an immunofluorescent-based technique that enables the detection of in situ protein-protein interaction by proximity, i.e., in a complex. This technique can be utilized to detect protein-protein interaction in fixed samples such as tissue sections, and we have previously utilized PLA to detect the formation of complex-IIb in MEFs [23]. Our data clearly demonstrates that TRADD and FADD are able to interact in multiple organs from Ripk1 ${ }^{-/}$animals at E18.5 (Fig. 5a, b). As FADD is the molecular bridge between TRADD (via death domain interactions) and caspase-8 (via death effector 
A

A
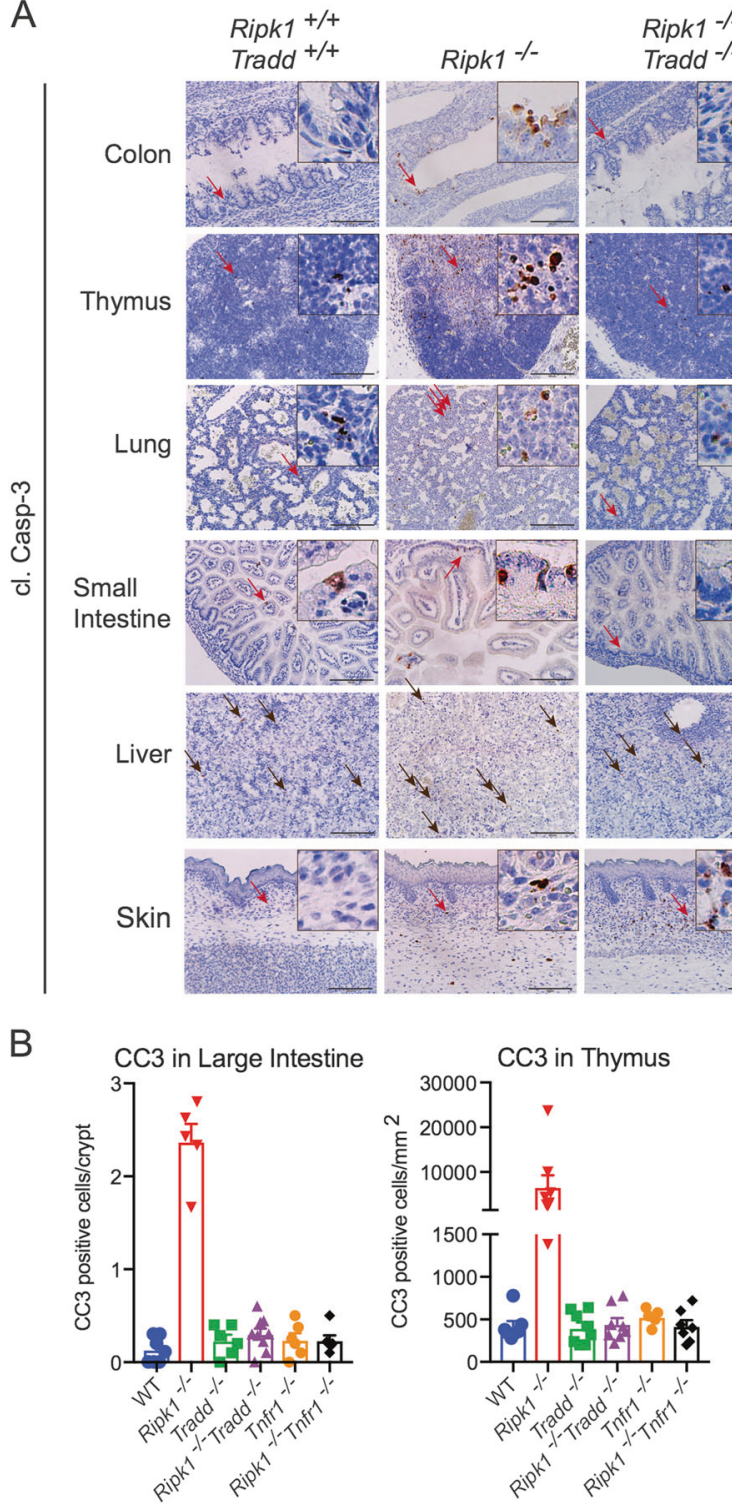

Fig. 3 Tradd Deficiency Rescues the Widespread Cleaved Caspase-3 Observed in Ripk1 ${ }^{-1-}$ Neonates and Reduces Systemic Cell Death. a Tissue sections of the indicated genotypes stained with CC3 (brown) and hematoxylin (blue). Each image is representative of results from at least three mice. Brown cells are positive for cleaved caspase-3. Red arrows indicate the region of tissue that has been magnified and brown arrows indicate cleaved caspase- 3 positive cells. b Quantification of cleaved caspase- 3 positive cells in the large intestine and thymus.

domain interactions), it is conceivable that the TRADD-FADD complex that we observed in Ripkl ${ }^{-1-}$ mice is also in a complex with caspase-8. Consistent with TRADD executing caspase- 8 activation outside of TNFR1, Ripk $1^{-/} \mathrm{Tnfrl}^{-/-}$mice displayed only a slight reduction in the TRADD-FADD complex in both liver and lung sections of E18.5 neonates (Fig. 5a). The colon, thymus and skin of Ripkl $^{-/}$mice also have considerable TRADD-FADD complex formation, which, unlike the liver

and lung, is dependent on TNFR1, as this complex is absent

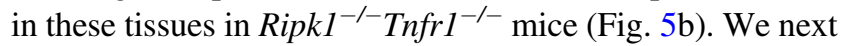

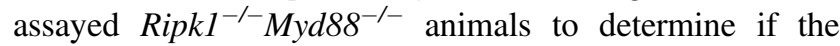
TNFR1-independent TRADD/FADD complex was Myd88 driven. We observed a clear reduction in TRADD/FADD complex in the lungs of Ripk $1^{-1-}$ Myd $88^{-/-}$animals, however, unexpectedly we observed an increase in TRADD/ FADD complex in the liver of Ripk $1^{-1-} M y d 88^{-/-}$animals (Figure S5A). 
A

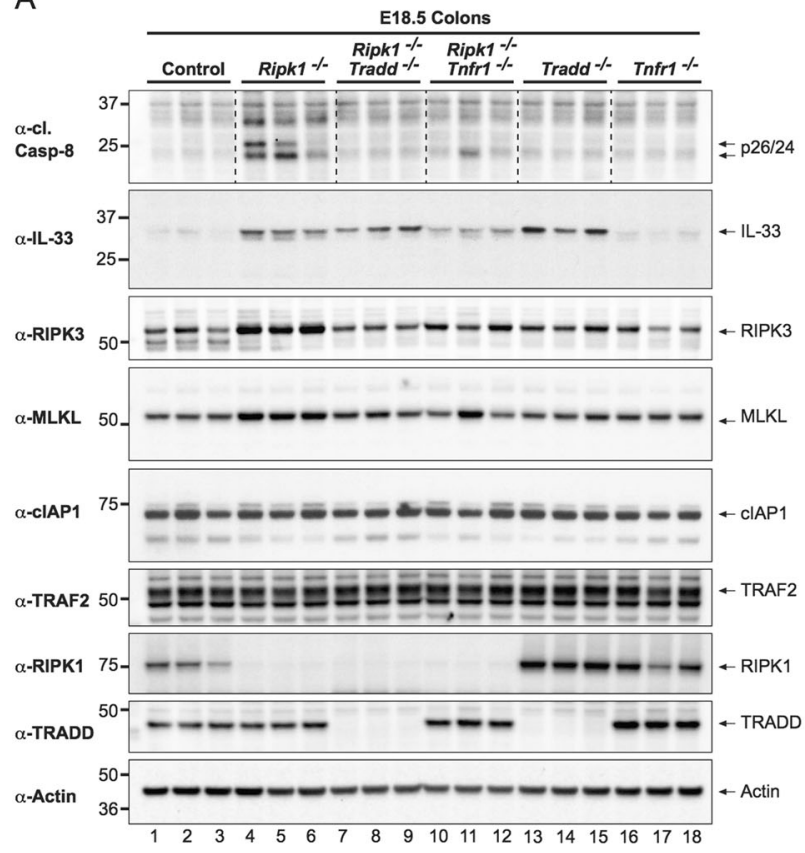

B

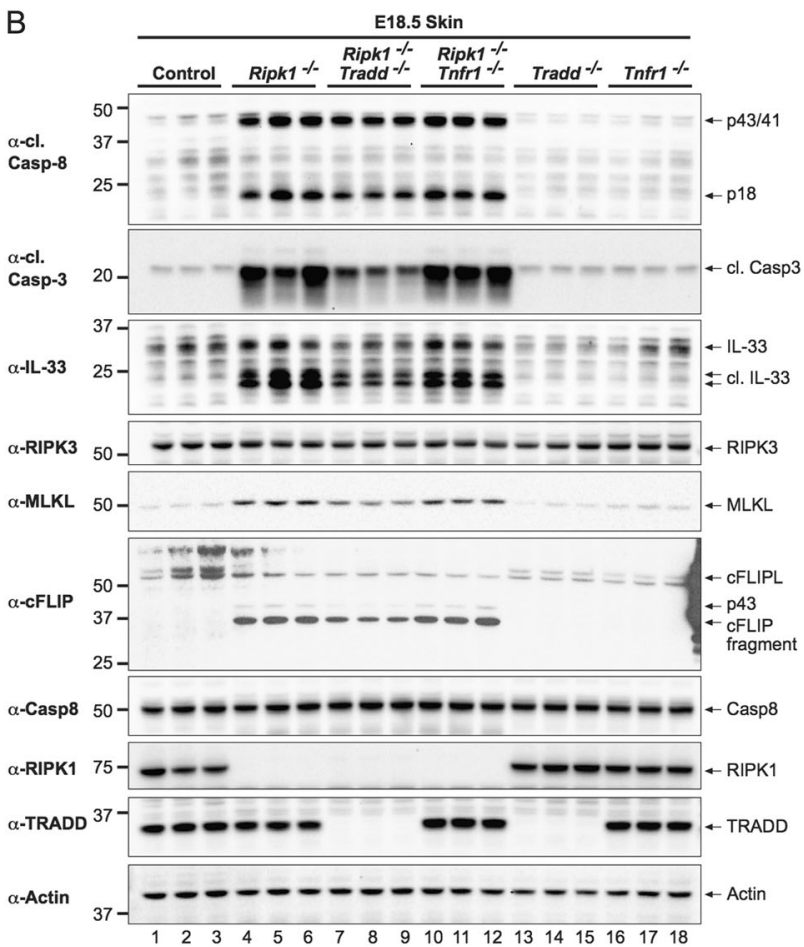

Fig. 4 Necroptotic and Apoptotic Markers are Reduced in Ripk1 ${ }^{-/-}$Tradd $^{-/-}$Neonate Tissues. Western blot analysis of colon or back skin lysates made from whole tissue taken from E18.5 embryos. Three embryos of each genotype were probed for the indicated proteins

\section{RIPK3 deletion rescues the prenatal lethality of Ripk1 ${ }^{-1-}$ Tradd $^{-1-}$ animals}

Given the similarity in phenotypes observed between our Ripk ${ }^{-1-}$ Tradd $^{-1-}$ neonates and the published Ripk1 ${ }^{-/}$Casp $^{-/-}$neonates [6-8] we reasoned that deletion of Ripk3 may provide a survival advantage to Ripk1 ${ }^{-/}$Tradd ${ }^{-/}$animals comparable to the protection seen in Ripkl ${ }_{--1}^{-1}$ radd $^{-/-}$Ripk $^{-/-}$mice. We, therefore, generated Ripk1 ${ }^{-/}$Tradd $^{-/-}$Ripk $^{-/-}$mice and observed a significant, albeit incomplete, extension of lifespan compared to Ripkl ${ }^{-/}$Tradd $^{-/-}$mice. Ripk ${ }^{-/-}$Tradd $^{-1-}$ Ripk $^{-/-}$mice survive between 22 and 49 days (Fig. 6a), are runty compared to littermate controls (Fig. 6b, c), have splenomegaly (Fig. 6d) and have no detectable payers patches (Fig. 6e). Histological analysis of $\operatorname{Ripk}^{-/-} \mathrm{Tradd}^{-/-} \mathrm{Ripk}^{-/-}$mice revealed disordered splenic architecture, however, in a panel of other tissues we observed no obvious histopathology (Fig. 6f). Of note, during the generation of our Ripk ${ }^{-1-}$ Tradd $^{-1-}$ Ripk3 ${ }^{-}-$mouse line our mouse facility identified Pasteurella infections in a number of mouse cages. Although the mice shown in this paper did not appear to exhibit macroscopic signs of Pasteurella infection we wish to highlight that this infection was confirmed present in our mouse room during the generation of the Ripk $I^{-/-}$Tradd $^{-/-}$Ripk $^{-/-}$mice and we suspect it may be present in the colony itself which could conceivably contribute to animal mortality.

\section{Discussion}

RIPK1 is a pro-survival molecule that plays a central role in maintaining tissue homeostasis during embryogenesis by preventing both apoptosis and necroptosis [6-8]. RIPK1 inhibits necroptosis and systemic inflammation by limiting the formation of a RIPK3-DAI RHIM mediated complex $[18,19]$. The involvement of RIPK1 in chronic intestinal inflammation has also placed RIPK1 as a central inhibitor of FADD-caspase-8 driven apoptosis [10], however, the molecule responsible for unleashing this cell death modality in vivo has remained elusive. Here we show that RIPK1 limits the activation of TRADD/FADD-mediated caspase- 8 activity and RIPK3 activity to maintain tissue homeostasis and ensure proper embryonic development (Fig. 6g). Ripk1 ${ }^{-/-}$Tradd $^{-/-}$neonates are fully rescued from the disrupted intestinal phenotype, thymic phenotype and anemia, and display reduced caspase cleavage in a broad range of tissues, when compared with Ripkl ${ }^{-/-}$embryos. Ripkl ${ }^{-\prime}$ Tradd $^{-/}$animals present with reduced systemic cell death and inflammation, however, they still die perinatally. Ripk ${ }^{-1-}$ Tradd $^{-1-}$ Ripk $^{-/-}$mice on the other hand survive weaning, however, we noted mortality between 22-49 days indicating that Ripk $^{-/-}$Tradd $^{-1}$ Ripk $^{{ }^{-1-}}$ mice are not comparable to Ripk ${ }^{-/}$Casp $^{-/-}$Ripk $^{-/-}$mice. Ripk ${ }^{-/-}$Tradd ${ }^{-/}{ }^{-}$ipk $^{-/-}$animals present with similar survival advantages to $\operatorname{Ripk}^{-/-} \mathrm{Tnfrl}^{-/} \mathrm{Ripk}^{-/-}$animals; however, 
Fig. 5 TRADD complexes with FADD in Ripk $1^{-1-}$ Neonates to Activate Caspase-8. a, b Tissue sections of indicated genotypes stained with anti-phalloidin-633 (red), DAPI (blue), and FADDTRADD complex (green) following PLA staining. Each image is a representative result from three mice
A

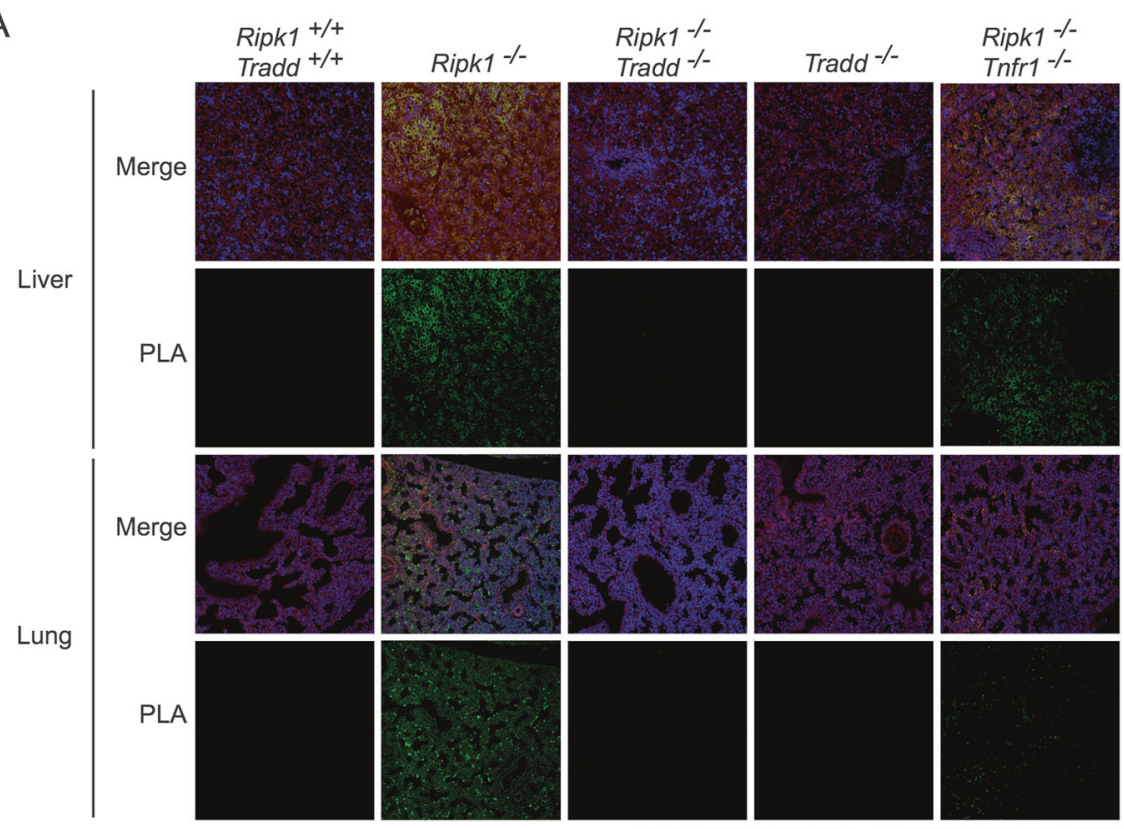

PLA:FADD/TRADD IF: PHALLOIDIN DAPI

B

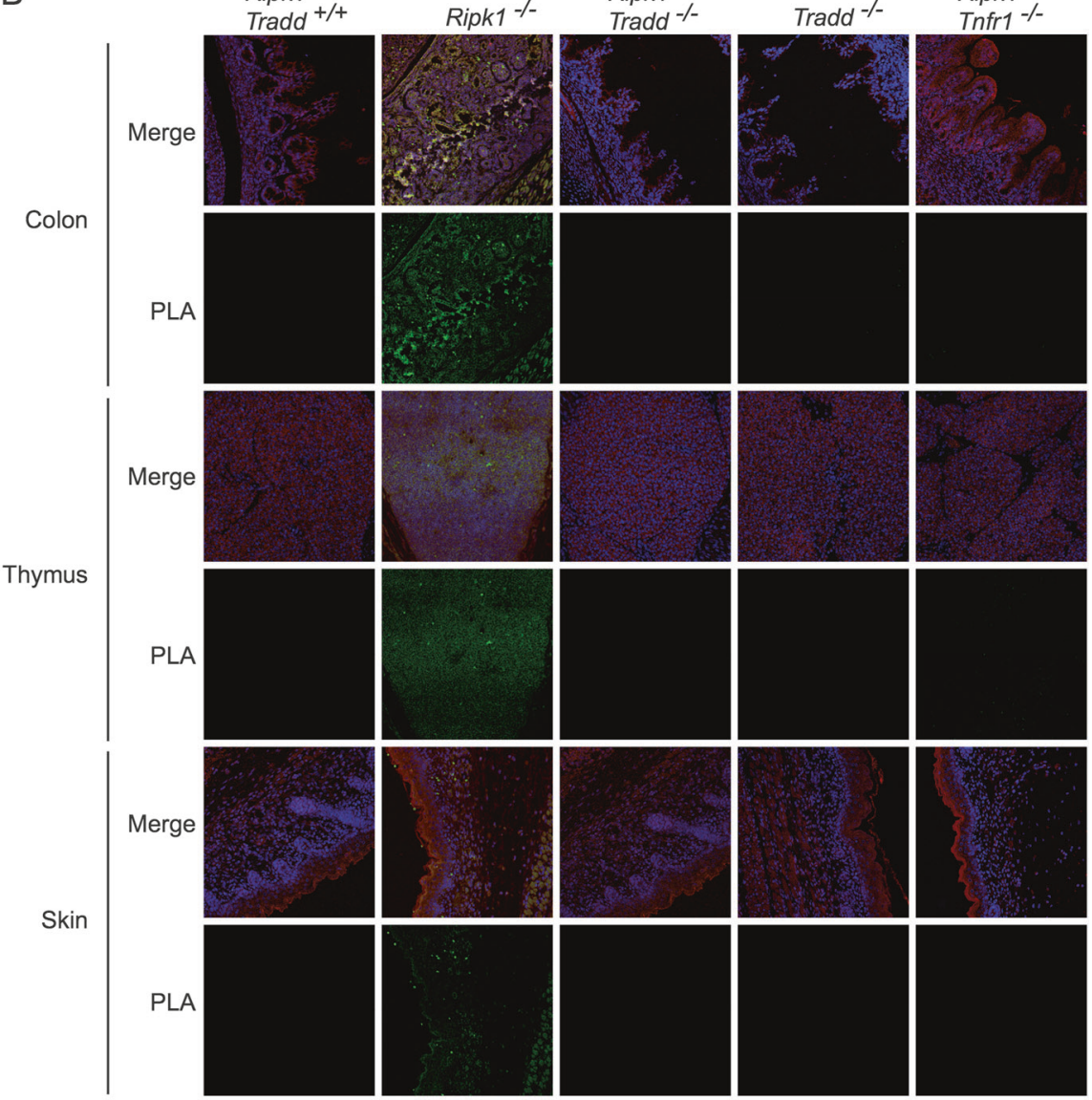

PLA:FADD/TRADD IF: PHALLOIDIN DAPI unlike Ripk1 ${ }^{-/-}$Tnfrl ${ }^{-/}{ }^{-}$ipk3 $^{-/-}$mice [6], Ripk1 ${ }^{-/-}$Tradd ${ }^{-/}{ }^{-}$ip $3^{-/-}$mice have largely normal colonic structure via H\&E staining. These genetic crosses strongly suggest that caspase- 8 can be activated in some tissues independently of TNFR1 and also TRADD in Ripk $1^{-/}$mice and that this contributes to the premature mortality observed in RipkI 

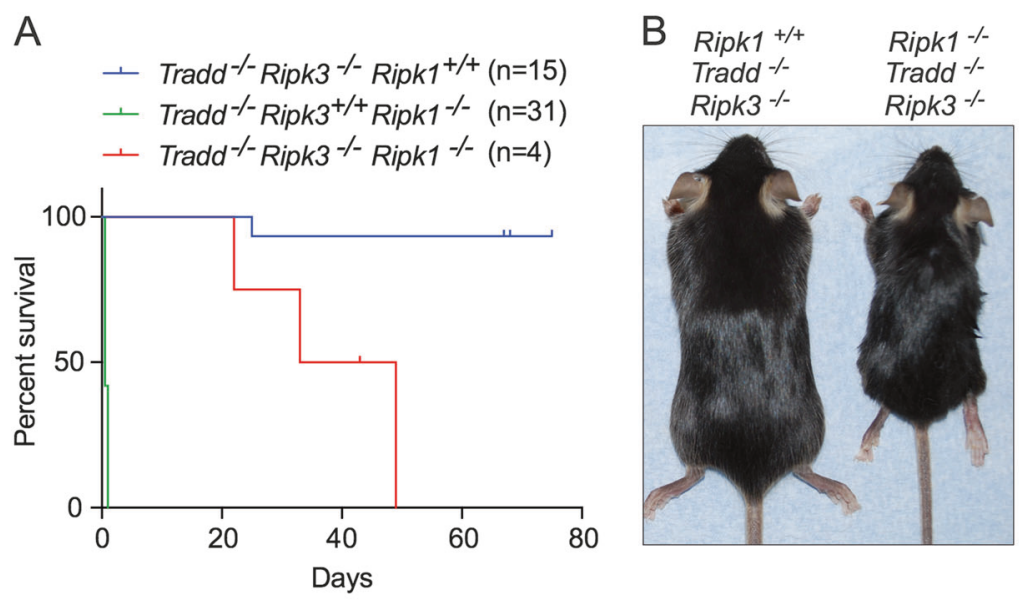

C

D

E
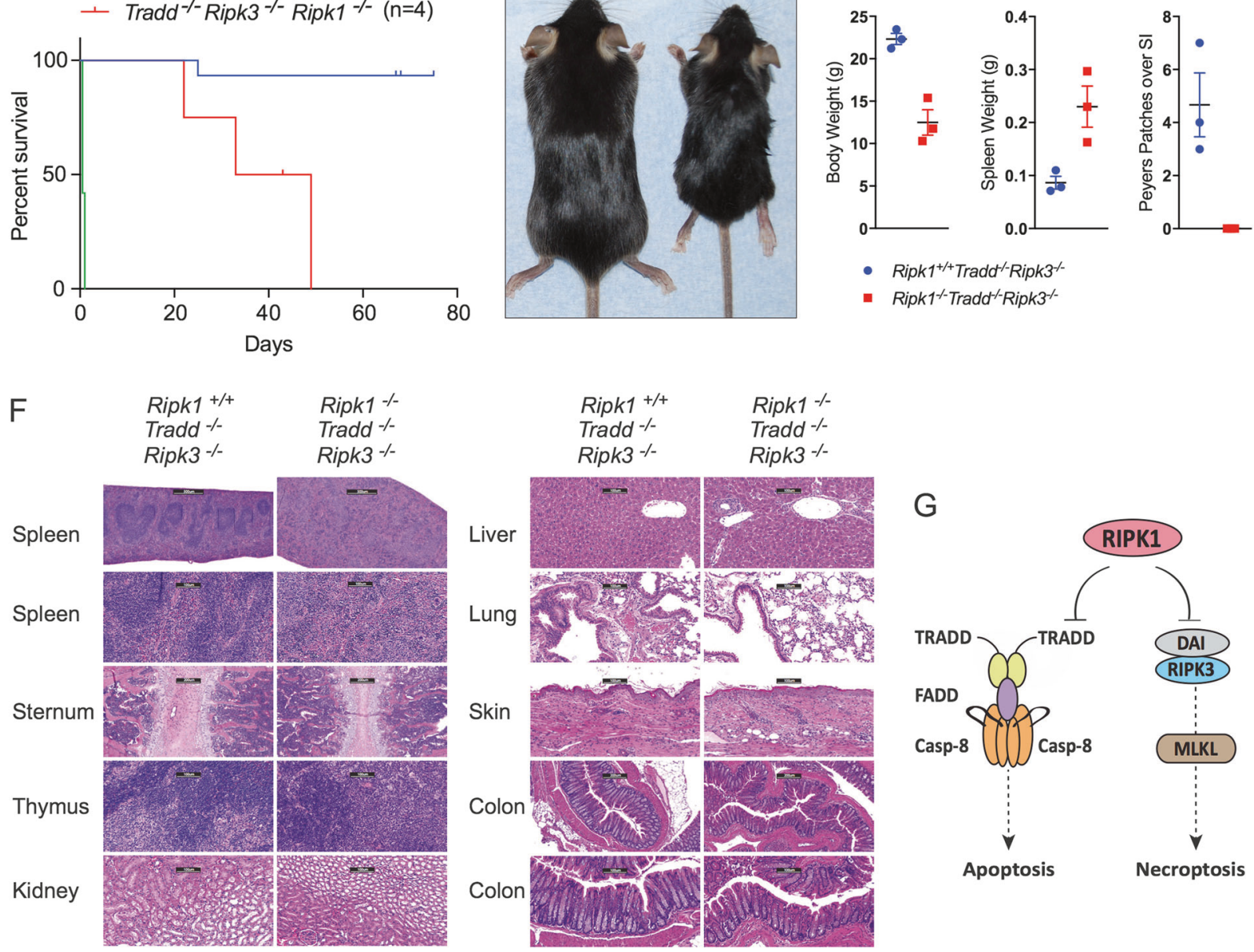

Fig. 6 RIPK3 deletion rescues the perinatal lethality observed in Ripk1 $1^{-/}$Tradd $^{-1}$ mice. a Survival curve for the indicated genotypes. b Images of mice from the indicated genotypes. $\mathbf{c}$ Total body weights of mice from the indicated genotypes. $\mathbf{d}$ Total spleen weights from the indicated genotypes. e Payers patches observed in small intestines (SI) of the indicated genotypes; counted macroscopically. f Tissue sections

of the indicated genotypes stained with hematoxylin and Eosin (H\&E). g Schematic depicting the important inhibitory role of RIPK1 for the prevention of TRADD/FADD-mediated caspase- 8 activation and RIPK3/DAI-mediated MLKL activation to maintain tissue homeostasis during development

${ }^{-/-}$Tnfr $^{-/-}$Ripk $^{-/-}$and Ripk1 ${ }^{-/-}$Tradd $^{-/-}$Ripk $^{-/-}$mice. This is consistent with our observation that caspase- 8 and caspase- 3 are still cleaved in the skin of Ripk1 ${ }^{-/}$Tradd $^{-1-}$ and $R i p k I^{-1-} \mathrm{TnFr}^{-1-}$ mice. Consistent with previous reports [8], Ripk1 ${ }^{-/-} \mathrm{Tnfrl}^{-/-}$animals had reduced multiorgan pathologies and in many ways phenocopied

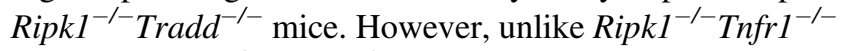
animals, Ripk1 ${ }^{-1-}$ Tradd $^{-1-}$ mice were no longer anemic; a Myd88-RIPK3 dependent phenotype that occurs independently of caspase-8 [8]. We observed that co-deletion of Ripk1 and Tradd was sufficient to rescue the reticulocyte defect, resulting in comparative reticulocyte numbers in Ripk1 ${ }^{-/}$Tradd $^{-/-}$neonates to their wild-type counterparts. This is suggestive of a restoration of bone marrow function in these animals, and that TRADD-mediated signaling is responsible for the reduction in reticulocytes in RipkI $^{-/-}$ mice. Tnfrl deletion was also sufficient to restore the reticulocyte numbers to normal, but was insufficient to rescue the anemia, suggesting that TRADD has essential roles outside of TNFR 1 signaling which are involved in driving anemia in Ripkl ${ }^{-/-}$animals. Given the requirement of Myd88 and RIPK3 to drive anemia in Ripk1 ${ }^{-/}$animals, our data also potentially places TRADD within a Myd88RIPK3 pathway in neonates, likely downstream of pattern recognition receptor-dependent processes, which are activated upon sterile inflammation and in which TRADD has described roles [29, 30, 35, 36]. In line with this, the levels of RIPK3, MLKL and the necroptotic DAMP IL-33 were altered in a TRADD dependent fashion in whole tissue from E18.5 neonates. The fact that Tradd or Tnfrl deletion 
phenocopy at the level of the reticulocyte defect suggests that an initial TNFR1-TRADD signal is initiated in Ripkl ${ }^{-/}$mice, likely at the level of the hematopoietic progenitors, resulting in a decrease in reticulocyte production. Consistently, bone marrow taken from Vav-iCre Ripk $1^{f l / f l}$ mice had a reduced propensity to form colonies when stimulated with TNF or Interferons ex vivo [33].

cFLIP is an important inhibitor of caspase- 8 whose role in vivo is complex and still incompletely understood. Knockout of cFLIP in mice $\left(\mathrm{Cflar}^{-1-}\right)$ results in embryonic lethality at E10.5 [37], which is rescued upon co-deletion of Ripk3 and Fadd and delayed upon deletion of Tnfr $[6,38]$. Conditional deletion of Cflar in intestinal epithelial cells results in TNFR1 driven perinatal lethality (P2) due to uncontrolled apoptosis [39]. Notably, cFLIP levels in whole intestinal lysates from Ripk1 ${ }^{-/-}$neonates (P0) are unchanged, whereas cFLIP levels within skin lysates from Ripkl ${ }^{-/}$neonates $(\mathrm{P} 0)$ are strongly up-regulated [8]. This is in contrast to our data, which shows that $\mathrm{CFLIP}_{\mathrm{L}}$ levels in the skin of Ripk1 ${ }^{-1-}$ neonates are decreased and which coincides with the appearance of a smaller cFLIP fragment occurring specifically in RipkI $^{-1-}$ animals. We currently do not understand the nature or the purpose of this smaller cFLIP fragment and we conclude that this is likely a cleaved product of cFLIP or a splice variant that occurs specifically in Ripk $^{-/-}$neonates. We also do not yet fully understand the discrepancy between our results and the previously published data on cFLIP levels. A possible explanation is that our embryos were harvested at E18.5; a full day earlier than previous studies [8]. This 1 day developmental window may alter the expression and regulation of cFLIP within neonates and would provide a plausible explanation for the difference in cFLIP levels.

Genetic evidence suggests that following RIPK1 deletion, RIPK1-independent necroptotic cell death is a source of DAMPs and causes sterile embryonic inflammation [40]. Caspase- 8 is not directly linked to the production of DAMPs as Ripk1 ${ }^{-/}$Casp $^{-/-}$mice are not protected from inflammation [40]. It was therefore surprising that the necroptotic DAMP IL-33 was reduced in RipkI ${ }^{-/-}$Tradd $^{-/-}$ neonates in both the skin and thymus, but not the colon. This indicates that TRADD-mediated signaling is required for the upregulation of inflammatory markers, such as IL-33, or that TRADD may directly alter the activities of RIPK3 and/or MLKL in a tissue specific manner. Unexpectedly, IL-33 was up-regulated in the colons of Tradd knockout mice, suggesting that TRADD may have an undescribed homeostatic function to prevent cell damage and DAMP release within the colon. Notably, we were unable to detect any changes in the phosphorylation status of MLKL in our tissue blots (data not shown) and, therefore, cannot reliably make any conclusions on the activation status of MLKL when Tradd is deleted.
While the evidence that TRADD can activate caspase- 8 in cellular systems is clear [36, 41], there is no evidence for this role of TRADD in vivo. A recent report has shown that loss of the NF-kappa-B essential modulator (NEMO) specifically within liver parenchymal cells $\left(\mathrm{NEMO}^{\mathrm{LPC}-\mathrm{KO}}\right)$ also induces the formation of complex-IIb, resulting in RIPK1 kinase-induced hepatocyte apoptosis and hepatocellular carcinoma. NEMO ${ }^{\mathrm{LPC}-\mathrm{KO}} \mathrm{RIPK} 1^{\mathrm{LPC}-\mathrm{KO}}$ mice still develop hepatocellular carcinoma and this can only be prevented by deletion of TRADD [42]. Differently, our data conclusively show that during embryogenesis RIPK1 prevents the interaction of TRADD with FADD thereby limiting aberrant activation of caspase-8. This event is partly driven via TNFR1, however, residual interaction in the liver and lung of Ripk1 $1^{-1}$ Tnfrl $^{-1-}$ mice suggests that cytosolic TRADD can still be recruited to FADD-caspase-8 independently of TNFR1. Consistent with this hypothesis, loss of Myd88-was sufficient to prevent the TRADD/FADD complex in the lung of Ripk1 ${ }^{-/-}$mice, however; conversely Myd88 loss increased the TRADD/FADD complex in Ripk1 ${ }^{-1-}$ livers. This not only suggests a direct involvement of TRADD in the activation of caspase- 8 , and consequent systemic cell death and inflammation, but also confirms a TNFR1independent contribution of TRADD to these pathophysiologies, and potentially demonstrates a role for Myd88 in limiting TRADD/FADD complex in the liver.

Our results demonstrate that RIPK1 has an important homeostatic function to limit the interaction of TRADD with FADD-caspase- 8 in the developing embryo and prevent apoptotic-mediated pathophysiologies. The mechanisms leading to the recruitment of TRADD to a caspase- 8 containing complex are still unclear. It is unlikely that the RHIM domain of RIPK1 is involved in inhibiting TRADD as mutation of the endogenous mouse RIPK1 RHIM domain caused RIPK3 dependent perinatal lethality [18, 19]. It is also unlikely that the TRAF2-cIAP1-RIPK1 axis is required to limit TRADD recruitment to caspase- 8 as both TRAF2 and cIAP1 levels remained unchanged in tissues where TRADD was responsible for the observed pathologies. Although speculative, it is plausible that RIPK1 merely has higher affinities for FADD compared to TRADD, and that TRADD can only fully reveal its function when the primary interacting molecule RIPK1, is removed.

\section{Experimental procedures}

\section{Mice}

All mice were backcrossed to C57BL/6J mice for $>10$ generations or generated on a C57BL/6J background. Mice were obtained by cesarean section at E18.5. Pregnant mice were prepared for cesarean delivery by progesterone 
injection at E17.5. The relevant Animal Ethics Committee approved all experiments.

\section{Histology and Immunofluorescence}

Embryos were fixed in $10 \%$ neutral buffered formalin, paraffin embedded, and sectioned for routine histology staining (H\&E). For skin immunofluorescence, paraffin sections were dewaxed, subjected to heat-induced epitope retrieval with citrate buffer then blocked and permeabilized with $1 \%$ BSA and $0.3 \%$ Triton X-100. IHC sections were stained with anti-CC3 (Cell Signaling Technology, 9661) or anti-Ki67 (Thermo Fisher Scientific, MA5-14520) and labeled polymer-HRP anti-rabbit secondary (Agilent Technologies K4011), or anti-CD45 (BD Pharmingen, BD553076) and goat anti-rat biotinylated secondary (Vector Laboratories, BA-9400). IF sections were stained with antiKeratin6 (Biolegend, 905701) or anti-Keratin14 (Biolegend, 905304) and goat anti-rabbit alexa-594 (Invitrogen, A-11012) or anti-CD11b conjugated to alexa 488 (Novus Biologicals, NB110-89474AF488). Samples were cover slipped using fluoromount with DAPI (ebioscience, 00-4959-52) enabling visualization of nuclei. Images were taken using a DP72 microscope and cellSens Standard software (Olympus).

\section{Cytokine bioplex assay}

The levels of 23 analytes were measured in mouse serum and protein lysate from E18.5 dorsal skin lysed in DISC lysis buffer, using a commercially available Bio-Plex Pro 23 Plex mouse Cytokine, Chemokine and Growth Factor Assay (Bio-Rad Laboratories Ltd, Hercules, CA, USA) on the Bio-Plex 200 System. Initial data analysis was undertaken using Bio-Plex Manager 5.0 Software to determine concentrations. Serially diluted standards ( $40 \mu \mathrm{l})$ and test serum, diluted 1 in 4 in sample diluent $(40 \mu \mathrm{l})$ and protein lysate $(500 \mu \mathrm{g} / 40 \mu \mathrm{l})$ was added to a plate containing magnetic antibody-coupled beads for each of the 23 analytes. The samples were incubated at room temperature on a plate shaker at 300 r.p.m. for $30 \mathrm{~min}$. Following washing with the Bio Plex Washing buffer, the secondary antibodies $(25 \mu \mathrm{l})$ were added to the plate and incubated as before. The plate was washed again and streptavidin-PE $(50 \mu \mathrm{l})$ was added and the plate incubated at room temperature on a plate shaker at 300 r.p.m. for $10 \mathrm{~min}$. Assay buffer $(125 \mu \mathrm{l})$ was added to each well of the plate before being analyzed on the Bio-Plex 200 machine. Fluorescent intensities obtained for the test samples were read from the standard curve to give $\mathrm{pg} / \mathrm{ml}$ values for each of the 23 analytes. Values below or above the reference range were assigned the value of the lowest or highest standard respectively.

\section{Immunoblotting}

Organ lysates were made by homogenizing colon or dorsal skin from E18.5 mice in ice-cold DISC lysis buffer containing complete protease inhibitor cocktail (Roche) and PhosSTOP (Roche), using a Tissue lyser 85300 (Qiagen). Lysates were clarified by centrifugation and supernatants were quantified with a BCA assay (Thermo Scientific). Supernatants were boiled with SDS reducing sample buffer. $3 \mu \mathrm{l}$ of plasma was diluted with $15 \mu \mathrm{l}$ of $5 \times$ SDS reducing buffer and boiled. Organ lysates (30 $\mu$ g of protein) and plasma ( $3 \mu \mathrm{l})$ were run on 4-12\% Bis-Tris gels (Invitrogen). Nitrocellulose (Plasma samples) or PVDF (all other samples) were probed with: CC 3 or -8 (Cell Signaling Technology 9661 and 8592, respectively), pro-caspase-8 (in house), IL33 (R\&D Systems AF3626), MLKL ([clone 3H1] described in Murphy et al. 2013), RIPK1 (BD Transduction 610458), RIPK3 (Axxora PSC-2283-c100), cIAP1 (Enzo [clone 1E1-1-10] ALX-803-335-C100), TRAF2 (Santa Cruz sc-876), TRADD (Santa Cruz [H-278] sc-7868), cFLIP (Cell Signaling 8510), or $\beta$-actin (Santa Cruz [I-19] sc-1616) antibodies.

\section{Hematology}

Automated cell counts were performed on blood collected from E18.5 embryos into tubes containing EDTA (Sarstedt) using an ADVIA 2120 hematological analyzer (Siemens). Blood smears were generated from 5 to $10 \mu$ l of blood, fixed with $100 \%$ methanol and stained with May-Grunwald Giemsa before microscopic examination. Images were acquired using a Nikon Eclipse E600 microscope, $\times 4 / 1.3$ NA or $\times 100 / 1.3$ NA oil objective with AxioCam Hrc and AxioVision 3.1 image acquisition software.

\section{PLA Assays}

Embryos were fixed in $10 \%$ neutral buffered formalin, paraffin embedded, and sectioned for routine histology. Paraffin sections were dewaxed, subjected to heat-induced epitope retrieval with citrate buffer then blocked and permeabilized with IFF buffer and $0.5 \%$ Triton X-100. Sections were then stained with anti-TRADD (Santa Cruz, sc7868) and anti-FADD (Santa Cruz, sc-6036) overnight at $4{ }^{\circ} \mathrm{C}$. Primary antibodies were washed $3 \times$ with $1 \times$ PBS, 0.1 $\%$ Triton X-100 for 10 mins. Proximity ligation assay was then performed as detailed by the manufacturer protocol with some alterations as follows. Secondary probes were left on the slides for $90 \mathrm{~min}$. Washes were performed in the humidity chamber at $37^{\circ} \mathrm{C}$. Ligation was performed for $40 \mathrm{~min}$ and amplification for $120 \mathrm{~min}$. Slides were then stained with anti-phalloidin-633 and DAPI (Invitrogen) for $1 \mathrm{~h}$. Slides were then washed $3 \times$ with 
PBS $0.1 \%$ Triton $\mathrm{X}-100$ for $10 \mathrm{~min}$ and $1 \times \mathrm{H}_{2} \mathrm{O}$. Slides were mounted with prolong gold antifade (Invitrogen) and images were acquired using the LSM719 Zeiss microscope, objective $\times 20$. Images were acquired by sequential scanning.

\section{Statistics}

Unless otherwise specified, data are presented as mean +1 SEM. Comparisons were performed using an unpaired nonparametric Mann Whitney test.

Acknowledgements We thank Pascal Meier for his support and acknowledge that the idea underlying this study was presented and discussed during a Meier lab retreat, we thank the staff in the WEHI Bioservices facility and Histology Department. We thank Michelle Kelliher for the Ripk $1^{-/-}$mice, Heinrich Korner for the Tnfr $1^{-/-}$mice and Tak W. Mak for the Tradd ${ }^{-1}$ mice [29]. This work was supported by National Health and Medical Research Project grants (1081272, 1046984, 1057888, 1101405, 1060179), fellowships to J.E.V. (1052598) and J.S 1107149, Independent Research Institutes Infrastructure Support Scheme Grant (9000220), a Victorian State Government Operational Infrastructure Support Grant and an Australian Government Research Training Program Scholarship and NHMRC scholarship to H.A (1093637). G.L. was supported by the ICR Dean Award and by Breast Cancer Now.

Author contributions Phenotypic analysis was performed by HA, RF, and JAR. Mouse crosses were established by JS, RF, JAR, and HA. Genotyping was performed by $\mathrm{CH}$. Histological and immunohistological analysis was performed by HA, RF, GL, and DS. Western blot analysis was performed by RF. PLA (Duolink) was performed by GL. EBS performed all Bioplex assays. A.P.N and L.D.R performed blood smears and polychromatic cell counts. JEV and JS provided reagents and advice and assisted in the interpretation of experiments. $\mathrm{RF}$ and GL conceived and coordinated the project, interpreted results, and wrote the manuscript.

\section{Compliance with ethical standards}

Conflict of interest The authors declare that they have no conflict of interest.

\section{References}

1. Vandenabeele P, Declercq W, Van Herreweghe F, Vanden Berghe T. The role of the kinases RIP1 and RIP3 in TNF-induced necrosis. Sci Signal. 2010;3:re4.

2. Meylan E, Burns K, Hofmann K, Blancheteau V, Martinon F, Kelliher M, et al. RIP1 is an essential mediator of Toll-like receptor 3-induced NF-kappa B activation. Nat Immunol. 2004;5: 503-7.

3. Thapa RJ, Nogusa S, Chen P, Maki JL, Lerro A, Andrake M, et al. Interferon-induced RIP1/RIP3-mediated necrosis requires PKR and is licensed by FADD and caspases. Proc Natl Acad Sci USA. 2013;110:E3109-18.

4. Rajput A, Kovalenko A, Bogdanov K, Yang SH, Kang TB, Kim JC, et al. RIG-I RNA helicase activation of IRF3 transcription factor is negatively regulated by caspase-8-mediated cleavage of the RIP1 protein. Immunity. 2011;34:340-51.
5. Tanzer MC, Khan N, Rickard JA, Etemadi N, Lalaoui N, Spall SK, et al. Combination of IAP antagonist and IFNgamma activates novel caspase-10- and RIPK1-dependent cell death pathways. Cell Death Differ. 2017;24:481-91.

6. Dillon CP, Weinlich R, Rodriguez DA, Cripps JG, Quarato G, Gurung P, et al. RIPK1 blocks early postnatal lethality mediated by caspase-8 and RIPK3. Cell. 2014;157:1189-202.

7. Kaiser WJ, Daley-Bauer LP, Thapa RJ, Mandal P, Berger SB, Huang C, et al. RIP1 suppresses innate immune necrotic as well as apoptotic cell death during mammalian parturition. Proc Natl Acad Sci USA. 2014;111:7753-8.

8. Rickard JA, O'Donnell JA, Evans JM, Lalaoui N, Poh AR, Rogers T, et al. RIPK1 regulates RIPK3-MLKL-driven systemic inflammation and emergency hematopoiesis. Cell. 2014;157: $1175-88$.

9. Dannappel M, Vlantis K, Kumari S, Polykratis A, Kim C, Wachsmuth L, et al. RIPK1 maintains epithelial homeostasis by inhibiting apoptosis and necroptosis. Nature. 2014;513:90-94.

10. Takahashi N, Vereecke L, Bertrand MJ, Duprez L, Berger SB, Divert $\mathrm{T}$, et al. RIPK1 ensures intestinal homeostasis by protecting the epithelium against apoptosis. Nature. 2014;513: 95-99.

11. Kelliher MA, Grimm S, Ishida Y, Kuo F, Stanger BZ, Leder P. The death domain kinase RIP mediates the TNF-induced NF-kappaB signal. Immunity. 1998;8:297-303.

12. Cusson N, Oikemus S, Kilpatrick ED, Cunningham L, Kelliher M. The death domain kinase RIP protects thymocytes from tumor necrosis factor receptor type 2-induced cell death. J Exp Med. 2002;196:15-26.

13. Upton JW, Kaiser WJ, Mocarski ES. Virus inhibition of RIP3-dependent necrosis. Cell Host Microbe. 2010;7:302-13.

14. Wong WW, Gentle IE, Nachbur U, Anderton H, Vaux DL, Silke J. RIPK1 is not essential for TNFR1-induced activation of NF-kappaB. Cell Death Differ. 2010;17:482-7.

15. Vanlangenakker N, Bertrand MJ, Bogaert P, Vandenabeele P, Vanden Berghe T. TNF-induced necroptosis in L929 cells is tightly regulated by multiple TNFR1 complex I and II members. Cell Death Dis. 2011;2:e230.

16. Moujalled DM, Cook WD, Okamoto T, Murphy J, Lawlor KE, Vince JE, et al. TNF can activate RIPK3 and cause programmed necrosis in the absence of RIPK1. Cell Death Dis. 2013;4: e465.

17. Zheng L, Bidere N, Staudt D, Cubre A, Orenstein J, Chan FK, et al. Competitive control of independent programs of tumor necrosis factor receptor-induced cell death by TRADD and RIP1. Mol Cell Biol. 2006;26:3505-13.

18. Lin J, Kumari S, Kim C, Van TM, Wachsmuth L, Polykratis A, et al. RIPK1 counteracts ZBP1-mediated necroptosis to inhibit inflammation. Nature. 2016;540:124-8.

19. Newton K, Wickliffe KE, Maltzman A, Dugger DL, Strasser A, Pham VC, et al. RIPK1 inhibits ZBP1-driven necroptosis during development. Nature. 2016;540:129-33.

20. Petersen SL, Wang L, Yalcin-Chin A, Li L, Peyton M, Minna J, et al. Autocrine TNFalpha signaling renders human cancer cells susceptible to Smac-mimetic-induced apoptosis. Cancer Cell. 2007;12:445-56.

21. Varfolomeev E, Blankenship J, Wayson S, Fedorova A, Kayagaki $\mathrm{N}$, Garg P, et al. IAP Antagonists Indu ceAutoubiquitination of c-IAPs, NF-kB activation, and TNFa-dependent apoptosis. Cell. 2007;131:669-81.

22. Vince JE, Wong WW, Khan N, Feltham R, Chau D, Ahmed AU, et al. IAP antagonists target cIAP1 to induce TNFalpha-dependent apoptosis. Cell. 2007;131:682-93.

23. Jaco I, Annibaldi A, Lalaoui N, Wilson R, Tenev T, Laurien L, et al. MK2 phosphorylates RIPK1 to prevent TNF-induced cell death. Mol Cell. 2017;66:698-710 e695. 
24. Girnius N, Davis RJ. TNFalpha-mediated cytotoxic responses to IAP inhibition are limited by the p38alpha MAPK pathway. Cancer Cell. 2016;29:131-3.

25. Lalaoui N, Hanggi K, Brumatti G, Chau D, Nguyen NY, Vasilikos $\mathrm{L}$, et al. Targeting p38 or MK2 enhances the anti-leukemic activity of smac-mimetics. Cancer Cell. 2016;29:145-58.

26. Dondelinger Y, Delanghe T, Rojas-Rivera D, Priem D, Delvaeye T, Bruggeman I, et al. MK2 phosphorylation of RIPK1 regulates TNF-mediated cell death. Nat Cell Biol. 2017;19: 1237-47.

27. Menon MB, Gropengiesser J, Fischer J, Novikova L, Deuretzbacher A, Lafera J, et al. p38MAPK/MK2-dependent phosphorylation controls cytotoxic RIPK1 signalling in inflammation and infection. Nat Cell Biol. 2017;19:1248-59.

28. Dondelinger Y, Aguileta MA, Goossens V, Dubuisson C, Grootjans S, Dejardin E, et al. RIPK3 contributes to TNFR1mediated RIPK1 kinase-dependent apoptosis in conditions of cIAP1/2 depletion or TAK1 kinase inhibition. Cell Death Differ. 2013;20:1381-92.

29. Chen NJ, Chio II, Lin WJ, Duncan G, Chau H, Katz D, et al. Beyond tumor necrosis factor receptor: TRADD signaling in toll-like receptors. Proc Natl Acad Sci USA. 2008;105: 12429-34.

30. Pobezinskaya YL, Kim YS, Choksi S, Morgan MJ, Li T, Liu C, et al. The function of TRADD in signaling through tumor necrosis factor receptor 1 and TRIF-dependent Toll-like receptors. Nat Immunol. 2008;9:1047-54.

31. Conrad M, Angeli JP, Vandenabeele P, Stockwell BR. Regulated necrosis: disease relevance and therapeutic opportunities. Nat Rev Drug Discov. 2016;15:348-66.

32. Wang L, Du F, Wang X. TNF-alpha induces two distinct caspase-8 activation pathways. Cell. 2008;133:693-703.

33. Roderick JE, Hermance N, Zelic M, Simmons MJ, Polykratis A, Pasparakis M, et al. Hematopoietic RIPK1 deficiency results in bone marrow failure caused by apoptosis and RIPK3-mediated necroptosis. Proc Natl Acad Sci USA. 2014;111:14436-41.

34. Gentle IE, Wong WW, Evans JM, Bankovacki A, Cook WD, Khan NR, et al. In TNF-stimulated cells, RIPK1 promotes cell survival by stabilizing TRAF2 and cIAP1, which limits induction of non-canonical NF-kappaB and activation of caspase-8. J Biol Chem. 2011;286:13282-91.

35. Ermolaeva MA, Michallet MC, Papadopoulou N, Utermohlen O, Kranidioti K, Kollias G, et al. Function of TRADD in tumor necrosis factor receptor 1 signaling and in TRIF-dependent inflammatory responses. Nat Immunol. 2008;9:1037-46.

36. Iordanov MS, Kirsch JD, Ryabinina OP, Wong J, Spitz PN, Korcheva VB, et al. Recruitment of TRADD, FADD, and caspase 8 to double-stranded RNA-triggered death inducing signaling complexes (dsRNA-DISCs). Apoptosis. 2005;10:167-76.

37. Yeh WC, Itie A, Elia AJ, $\mathrm{Ng} \mathrm{M}$, Shu HB, Wakeham A, et al. Requirement for Casper (c-FLIP) in regulation of death receptorinduced apoptosis and embryonic development. Immunity. 2000; 12:633-42.

38. Dillon CP, Oberst A, Weinlich R, Janke LJ, Kang TB, Ben-Moshe $\mathrm{T}$, et al. Survival function of the FADD-CASPASE-8-cFLIP(L) complex. Cell Rep. 2012;1:401-7.

39. Piao X, Komazawa-Sakon S, Nishina T, Koike M, Piao JH, Ehlken $\mathrm{H}$, et al. c-FLIP maintains tissue homeostasis by preventing apoptosis and programmed necrosis. Sci Signal. 2012;5:ra93.

40. Silke J, Rickard JA, Gerlic M. The diverse role of RIP kinases in necroptosis and inflammation. Nat Immunol. 2015;16:689-97.

41. Hsu H, Shu HB, Pan MG, Goeddel DV. TRADD-TRAF2 and TRADD-FADD interactions define two distinct TNF receptor 1 signal transduction pathways. Cell. 1996;84:299-308.

42. Kondylis V, Polykratis A, Ehlken H, Ochoa-Callejero L, Straub BK, Krishna-Subramanian S, et al. NEMO prevents steatohepatitis and hepatocellular carcinoma by inhibiting RIPK1 kinase activitymediated hepatocyte apoptosis. Cancer Cell. 2015;28:830. 